\title{
Rerepresenting Autonomated Vehicles in a Macroscopic Transportation Model
}

\author{
Árpád Török*, Zsolt Szalay', Gábor Uti², Bence Verebélyi² \\ ${ }^{1}$ Department of Automotive Technologies, Faculty of Transportation Engineering and Vehicle Engineering, \\ Budapest University of Technology and Economics, H-1521 Budapest, P.O.B. 91, Hungary \\ 2 RelativeGAP, Lingfield, Surrey, 5 Greenwich View Place, London, E14 9NN, United Kingdom \\ * Corresponding author, e-mail: arpad.torok@auto.bme.hu
}

Received: 08 March 2019, Accepted: 09 April 2019, Published online: 31 October 2019

\begin{abstract}
The main goal of this article is to determine a comprehensive and well applicable model architecture, which is adequate to estimate the system level advantages with regard to automated transportation and which is appropriate to determine possible costs and losses with regard to the approach of such transport modes. In the study the Budapest Transportation Model is applied. Taking autonomous vehicle penetration into account as an external variable, in the analysis a constant growth is assumed in the penetration of automated vehicles. This article has taken the most relevant factors of transportation network into account with regard to automated cars. It is also important to mention that the paper presents the most important modelling phases, where automated cars can be taken into account during the macroscopic modelling process. In the first step of the process during the network definition phase it is possible to consider the effect of automated vehicles on the transport system (e.g. separated routes). The next phase where the effect of automated vehicles should be taken into consideration is the mode choice step (e.g. different demand segments). And finally traffic assignment step, where the effect of automated vehicles can be represented. The easiest way for this is the modification of passenger car units through the parameter of assigned traffic per capacity ratio.
\end{abstract}

Keywords

automated cars, transportation demand, traffic analysis, impact assessment

\section{Introduction}

\subsection{Literature review, initial considerations}

In recent days, there is a significant development in the automotive domain, which affect the traditional transportation system significantly (Zhao et al., 2018). The role of automation plays a more and more important role with regard to vehicles systems (Tettamanti et al., 2016). The advantages of automated vehicles involve more efficient, sustainable and safer transportation (Zöldy, 2018). It is predicted, that autonomous transportation results in more reasonable mobility demand structures in the field of good and passenger transportation (Fiori et al., 2019). A comprehensive analysis have been presented by the authors to analyze the assumed effect of some relevant intervention (Török et al., 2019). In that article, cyberattacks are expected to target the transportation system and not the individual drivers. To represent the impact of the analyzed interventions the Budapest transport model has been applied. The analysis has been provided output related to the performance of the system with regard to the tested attacks (Kröger et al., 2018).

Due to the growing importance of automated cars with regard to the producer and the user groups as well, it is more and more relevant to consider the network effect of automated transport system.

Many professionals predict more automated vehicles. However these trends are significantly affected by relevant factors, it can be concluded that these market processes cannot be stopped (Fox-Penner et al., 2018). Other studies have predicted that a significant load on the electrical network can approach in the next few years due to the growing number of electric vehicles. On the other hand probably it is possible to satisfy the demands by the existing capacity (Farhan et al., 2018). Other models has proved that the economic efficiency of car-sharing systems can be provided if the proportion can achieve the $10 \%$ level (Ye et al., 2018). Relevant researches have investigated the 
impact of automated vehicles in case of traditional transport environment. The efficiency improvement and the developed saturation indicators have been validated by the research (Iacobucci et al., 2018). There are also relevant results in the field of economic modelling related to automated transport systems, presenting that these novel solutions (e.g. in Tokyo) can substitute more than 7 cars (Iacobucci et al., 2018).

This study is based on the Budapest Transportation model (Fig. 1). ${ }^{1}$ During the assessment, a constant growth has been taken into account with regard to the number of automated vehicles. So, the evaluated alternatives are investigated according to the penetration of automated vehicles. Since the driving assistant systems are assumed to develop continuously, reachable traffic density is estimated to increase significantly. The increasing traffic density has been derived from the modal-split of automated vehicles, because the existence of man-driven vehicles makes it necessary to consider perception and intervention time. Furthermore the research has not taken the growth in the number of un-reserved parking places into account and the cost savings with regard to decrease in traffic peaks derived from the more efficient parking processes. The introduced reduction related to the complexity of the model will lead to minor the achievable social-economic savings which support the main approach of the study to rather provide a careful estimation framework.

The assessment has been performed by a general transportation model framework, constructed from trip matrixes and a graph based network representation module. The trip matrixes describe the origin-destination data of the analyzed user groups, and the network module is built-up in the form of a directed graph. To examine the pre-defined alternatives, the outputs of the general transport model have been used (e.g. reduction in travelled hours, increase in travel speed, etc.). On the other hand it has been a core objective of the analysis to provide substantial consequences related to automotive engineering perspective with regard to the unit effect of the assumed automation. So, the final achievements of the evaluation is the analysis of a nowadays transportation framework and an automated system considering the city of Budapest.

So in the following part of the discussion the applied approaches are described. After that the obtained results are going to be outlined. In the final part of the paper the most important findings are explained.

1 prepared by the Centre for Budapest Transport

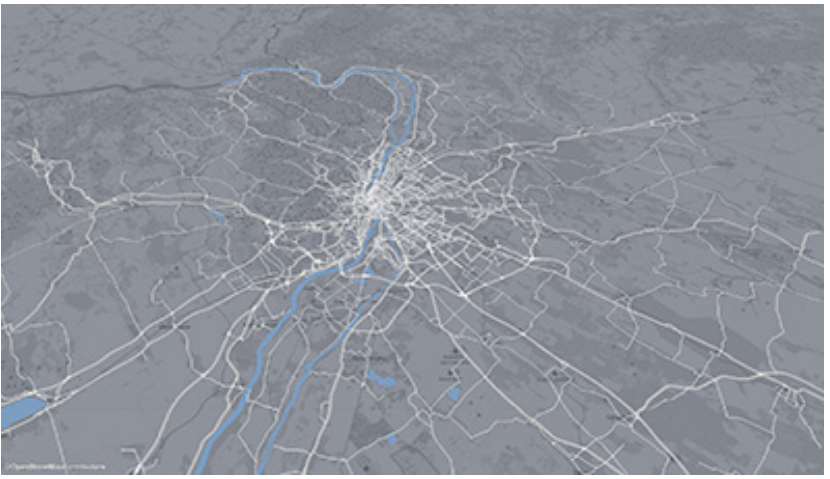

Fig. 1 Representation of the Budapest model

\section{Applied model structure}

The structure of the analysis is built up from the following parts: describing the involved methodological solution and identifying the input parameter-set definition of model parameters representing the applied assumptions.

The Transportation Model of Budapest ensured the methodological solution. The methodological solution has been implemented in PTV Visum framework. There are more than nine hundred traffic inner districts (Fig. 2), more than two hundred suburb districts and more than twenty districts outside the city. More than one and a half million travels are analyzed in the evaluation, which represents an ordinary day. The graph is constructed from more than ten thousand points and more than thirty thousand sections. The mobility activities have been examined by the equilibrium approach.

The used software defines the equilibrium state of the traffic structure according to Wardrop's first rule (Wardrop, 1952). This method determines the assigned traffic by programing the travels to the links of the network. The aim of the programing is to find new routes with shorter travel time. If another faster route can be found, the software can allocate travels to the other route. This programming step modifies the travel time of the

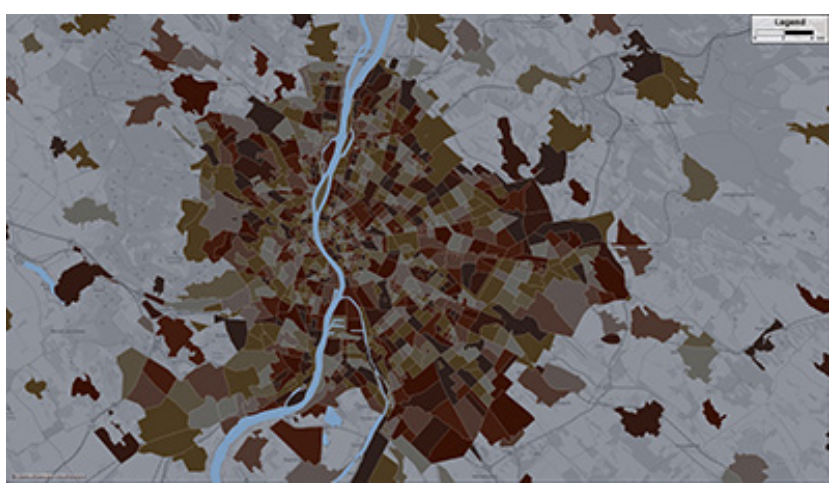

Fig. 2 Zone structure of the applied model 
involved routes, so the traffic structure is reorganized by the program. The program is ended when a balance in the traffic structure is set (Piątkowski et al., 2013). The previously interpreted calculation process is defined as the traffic assignment step, which is responsible for ordering journeys to the links of the graph. This process supports the derivation of travel data (e.g. time, length, etc.).

The traffic is assigned on the network through the following minimization process.

$$
M I N_{-} F=\sum_{k=1}^{n} \int_{0}^{t_{i}} R_{i}(x) d x
$$

Where $F$ : is sum of impedance like functions characterizing the i network component, $R_{i}(x)$ : is the impedance of network component $i$ with $x$ traffic, $t_{i}$ : traffic of network component $i$.

The introduced objective function represents the sum of traffic on the network with regard to the traffic of all investigated network components $\left(t_{i}\right)$ in case of all $i$. The constraint of the problem is defined based on the traffic going through a given point of the network. Accordingly, the sum of traffic to a specific point is the same as the sum of traffic leaving the given point. The goal of the optimization is to find the minimum of the sum of impendency values $R_{i}(x)$ with regard to the external variable of CAV saturation modifying parameter.

Section 3 tries to sum up the parameters, which should be considered during the evaluation of the saturation effect of automated vehicles (Friedrich et al., 2016).

As it is observable, Fig. 3 introduces the traditional modelling approach. Based on the represented original process, the novel methodological components and the developed modelling modules can be described.

First of all in the first step of the process during the network definition phase it is possible to consider the effect of automated vehicles on the transport system (Fig. 4). The reason for this, is assumption that in some cases the automated traffic might be channelized into dedicated corridors. This can make it necessary to separate these dedicated routes for connected and autonomous vehicles.

The next phase where the effect of automated vehicles should be taken into consideration is the mode choice step. The reason for this is the evaluation of transition periods in which case a mixed traffic can be expected, where traditional and automated vehicles can be expected to be operated on the road at the same time. This expectation could make it necessary to represent traditional and automated vehicle related demands in separated matrixes.

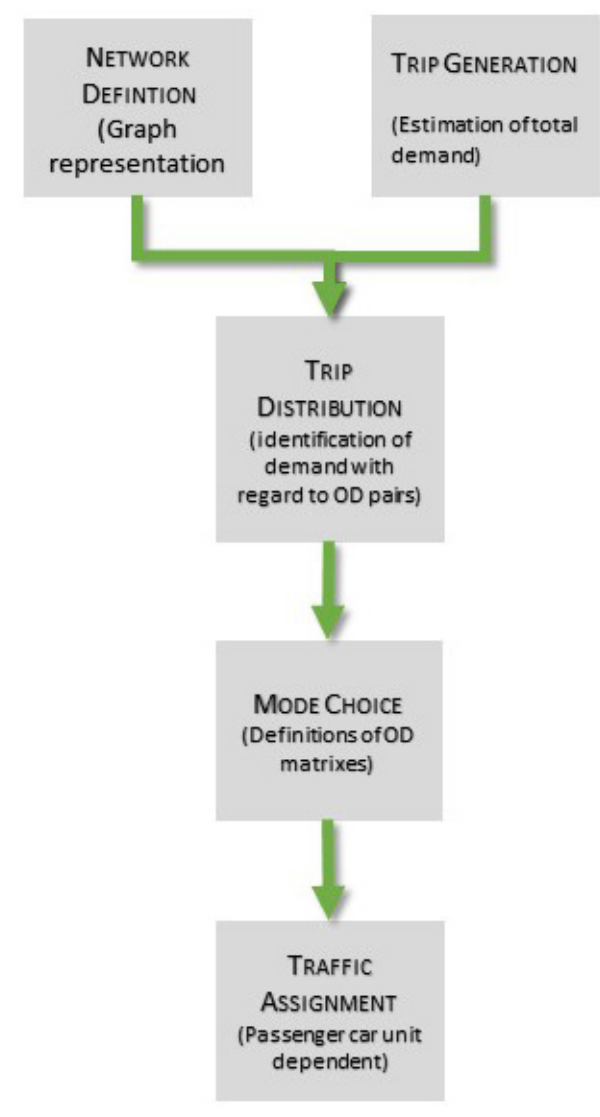

Fig. 3 Traditional traffic modelling approach

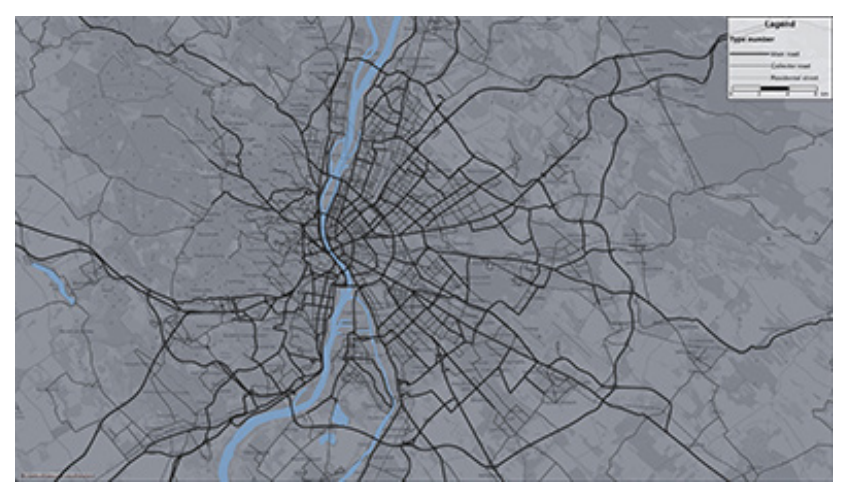

Fig. 4 Core network of the applied model

And finally traffic assignment step, where the effect of automated vehicles should be considered. The easiest way for this is the modification of passenger car units through the parameter of assigned traffic per capacity ratio. In the next steps this simplified representation is going to be discussed in details.

The distance between the successive vehicles is crucial related to the load factor of the graph links. The distance between the vehicles can be estimated based on the assumed speed, reaction and brake time (Hartmann et al., 2018; Rafat et al., 2019; Schramm et al., 2018). In case of 
human drivers, stopping distance consists of reaction distance and braking distance. Reaction distance is composed of further components: mental processing time, sensation time, perception time, response selection time, movement time (Green et al., 2000). The analyzed researches related to braking have proved that the process of decreasing the velocity is a kind of response, since mostly the velocity reduction is performed when dangerous event or factor has been recognized. Beside this, it also reasonable to be clarified that smaller the distance between the vehicles, the less load will appear in the given network element influencing the saturation of the whole network.

In the next step it seems to be unavoidable to determine those time components from the above interpreted brake process, that are advantageously influenced by an automated system elements.

To continue this thinking, it seems to be reasonable that velocity reduction process is going to be influenced also by external events or factors. This means that it is going to be a kind of responsive process. The reason for this is that a dangerous event or factor has to be started with the recognition, and then can the automobile provide the adequate response for the given event or factor. The automated function of velocity reduction could result a reasonable decrease in the value of the average stopping distance. This assumption could be supported by the expectation that automated functions could subtract the effect of human reaction from transportation processes. Furthermore, it can be clarified, that this development phase can be improved further. The reason for this is the future possibility of connected vehicles, which can provide solutions to organize velocity reduction processes based on preliminarily shared, highly reliable information, getting around the responsive characteristic of the velocity reduction process (Marilisa et al., 2018; Martin et al., 2017; Mikušová et al., 2018; Rizvi et al., 2017).

When we assume that the automobiles of the investigated system communicate with each other, velocity reduction can be expected to be transformed into an efficiently predictable, safe and secured process in most of the cases. The reason for this is the immediately available set of information shared for example by the leading automobile of a platoon in case of an accident. Accordingly, the new features of automated vehicles are expected to cause significant improvement in operation efficiency on a network level.

The introduced reasons have made us interpret the expectable impacts of the development phases in separated steps. So the automation phases are represented according to their expected impact on network saturation in a system level.

Due to the previously provided interpretation, it is reasonable to start separation with the factors influencing network saturation in a crucial way. To do this, the relevant system parameters have to be linked to the affecting automated functions.

After performing the mentioned analysis, the next parameters are found to be crucial from the viewpoint of the effectiveness of network:

- the relationship of assigned traffic and capacity on a network element,

- secondary travel time components (walking, parking).

According to the above presented considerations, the most important efficiency parameters (e.g. traffic per capacity ratio of the network) have been applied in the model in the light of automated and normal vehicle ratio. So the saturation factor has been defined in light of the assumed proportion of automated vehicles ordered to a given SAE level.

Assigned traffic per capacity ratio (TCR) is assumed to influence the occupied road length of vehicle unit, applied to describe the elemental parts of the traffic flows.

Based on the reviewed literature it has been become clear that the deviation of TCR expected value is reasonably significant if automated transportation become the ruling transport mode. The maximum capacity improvement can be beyond 5 times larger than the baseline value while the minimum value can be somewhere beyond 10 percent perceived capacity extension. Accordingly, TCR value is considered to be reduced by $35 \%$.

Numerous studies have been proved that secondary travel time including parking and walking can be about averagely 10 minutes related to a single trip in a city (Benenson et al., 2008; Shoup, 2006).

Summing up, based on the reviewed literature, automated transportation can averagely result in $35 \%$ decrease of TCR and over 10 minutes time saving at the most. However this result can be achieved by the complete penetration of fully automated vehicles, so the current level of automation is assumed to be the baseline state, so it is assumed to have no effect on the system.

\section{Outcomes}

The performed investigation has been based on the assessment of the following values: sum of travel time changes, sum of traffic volume changes and change in average 
speed and travel time values. Basically, the introduction of automated transportation should be evaluated depending on the penetration of the vehicle clusters characterized by different SAE levels. According to the most extreme opinions, if all the vehicles are autonomous, the time distance between the vehicles can be reduced to one second. This can lead to a significant reduction in TCR value, where the decrease can achieve the value of $60 \%$. However it has to be clarified that the expected impact of the automation processes should be separately investigated in case of the city center and the agglomeration, since the typical saturation and demand characteristics of the mentioned model areas are reasonably different.

According to the performed analysis it can be concluded that the expectable benefits of such a system development process can provide proper financial background for a major national level project.

\section{Explanation}

In Section 4 the evaluation possibility of the parameter set is interpreted (Jankowska et al., 2015; Sipos, 2017). It is needed to be mentioned that the objective evaluation would make it necessary to calculate with system costs as well. Beside this, it seems to be obvious that the larger the proportion of automated vehicles is the more effective the system can be, so this conclusion verifies the consideration to support the spread of automated vehicles as strongly as possible (Madleňák et al., 2018; Rosenberger et al., 2018; Zöldy et al., 2017).

As the result of the analysis presents, sum of travel time changes in case of a small-scale proportion of automated vehicles can be over ten thousand. And this result is very promising since this value can explain a major national level project.

It can be observed that the growing proportion of automated vehicles would the system-scale change of traveled vehicle kilometers behaves similarly to the sum of travel time changes. Although, it has to be mentioned that in the town-center the value of change in traveled vehicle kilometers is lower than the sum of travel time changes, since the length of journeys is typically much shorter in the town center. Summing up, it can be concluded that a small-scale proportion of automated vehicles can already cause two minutes travel time decrease. The large-scale spread of automated cars can cause over seven minutes average travel time decrease.

\section{Conclusion}

The main scientific contribution of our research is to provide a well-applicable modelling process structure, which can be a proper tool to collect the expected effects of the appearance of automated cars on our urban roads. Beside this, the method can also be used to estimate the negative effects of such a system (Buttyán et al., n.d.; Checkoway et al., 2011; Szíjj et al., 2015).

Beyond the summarized results, it is also important to highlight that the paper presents the most important modelling phases, where automated cars can be taken into account during the macroscopic modelling process. In the first step of the process during the network definition phase it is possible to consider the effect of automated vehicles on the transport system (e.g. separated routes). The next phase where the effect of automated vehicles should be taken into consideration is the mode choice step (e.g. different demand segments). And finally traffic assignment step, where the effect of automated vehicles can be represented. The easiest way for this is the modification of passenger car units through the parameter of assigned traffic per capacity ratio.

The model has been developed in the official framework of Budapest Macroscopic model based on a PTV VISUM environment. Automated vehicles have been taken into account during the representation phase by TCR parameter and secondary travel times. Beside this, model alternatives have been differentiated based on the assumed proportion of automated vehicles.

The further research has been based on the consideration that the expected impact of the automated car penetration is strongly influenced by the SAE levels of the vehicles. Accordingly, in the further steps of the research the applied input parameters have been TCR and the secondary travel time reduction. As mentioned before, during the investigation four main output indicators have been applied: sum of travel time changes, sum of traffic volume changes and change in average speed and travel time values. The result of our analysis can be briefly summarized by the amount of expected change in total travel during the investigated fifteen years long operation period, which can achieve the two billion value. Beside this the average duration of a journey can be decreased by thirty percent.

This study has analyzed the main factors of the mobility process with regard to automated road vehicles although 
there are other questions discussed new transport modes which cannot be left out of consideration in the next steps of the further development. As an outstandingly important research orientation, possible effects of cyber-attack have to be investigated in complex and thorough way.

\section{References}

Benenson, I., Martens, K., Birfir, S. (2008) "PARKAGENT: An agentbased model of parking in the city", Computers, Environment and Urban Systems, 32(6), pp. 431-439. https://doi.org/10.1016/j.compenvurbsys.2008.09.011

Buttyán, L. (n.d.) Cyber security of vehicles - the attack surface, Budapest University of Technology and Economics, Hungary.

Checkoway, S., McCoy, D., Kantor, B., Anderson, D., Shacham, H., Savage, S., Kohno, T. (2011) "Comprehensive experimental analyses of automotive attack surfaces", In: USENIX Security Symposium, San Francisco, CA, USA, pp. 447-462.

Farhan, J., Chen, T. D. (2018) "Impact of ridesharing on operational efficiency of shared autonomous electric vehicle fleet", Transportation Research Part C: Emerging Technologies, 93, pp. 310-321. https://doi.org/10.1016/j.trc.2018.04.022

Fiori, C., Arcidiacono, V., Fontaras, G., Makridis, M., Mattas, K., Marzano, V., Thiel, C., Ciuffo, B. (2019) "The effect of electrified mobility on the relationship between traffic conditions and energy consumption", Transportation Research Part D: Transport and Environment, 67, pp. 275-290. https://doi.org/10.1016/j.trd.2018.11.018

Fox-Penner, P., Gorman, W., Hatch, J. (2018) "Long-term U.S transportation electricity use considering the effect of autonomous-vehicles: Estimates \& policy observations", Energy Policy, 122, pp. 203-213. https://doi.org/10.1016/j.enpol.2018.07.033

Friedrich, B. (2016) "The effect of autonomous vehicles on traffic", In: Autonomous Driving, Springer, Berlin, Heidelberg, Germany, pp. 317-334.

https://doi.org/10.1007/978-3-662-48847-8_16

Green, M. (2000) "“How Long Does It Take to Stop?” Methodological Analysis of Driver Perception-Brake Times", Transportation Human Factors, 2(3), pp. 195-216. https://doi.org/10.1207/STHF0203_1

Hartmann, M., Abaunza, H., Castillo, P., Stolz, M., Watzenig, D. (2018) "Pedestrian in the loop: An approach using flying drones", In: 2018 IEEE International Instrumentation and Measurement Technology Conference (I2MTC), IEEE, Houston, TX, USA, pp. 1-6. https://doi.org/10.1109/I2MTC.2018.8409536

Iacobucci, R., McLellan, B., Tezuka, T. (2018) "Modeling shared autonomous electric vehicles: Potential for transport and power grid integration", Energy, 158, pp. 148-163. https://doi.org/10.1016/j.energy.2018.06.024

Jankowska, D., Mikušová, M., Wacowska-Ślęzak, J. (2015) "Mobility Issues in Selected Regions of Poland and Slovakia-Outcomes of International Project SOL (Save Our Lives) Survey", Periodica Polytechnica Transportation Engineering, 43(2), pp. 67-72. https://doi.org/10.3311/PPtr.7580

\section{Acknowledgements}

The research reported in this paper was supported by the Higher Education Excellence Program of the Ministry of Human Capacities in the frame of Artificial Intelligence research area of Budapest University of Technology and Economics (BME FIKP-MI/FM).

Kröger, L., Kuhnimhof, T., Trommer, S. (2018) "Does context matter? A comparative study modelling autonomous vehicle impact on travel behaviour for Germany and the USA", Transportation Research Part A: Policy and Practice, 122, pp. 146-161. https://doi.org/10.1016/j.tra.2018.03.033

Madleňák, R., Madleňáková, L., Hoštáková, D., Drozdziel, P., Török, A. (2018) "The Analyses of the Traffic Signs Visibility during Night Driving", Advances in Science and Technology Research Journal, 12(2), pp. 71-76. https://doi.org/10.12913/22998624/92103

Marilisa, B., Luigi, P., Nicola, B. G. (2018) "C-ITS communication: an insight on the current research activities in the European Union", International Journal of Transportation Systems, 3, pp. 52-63. [online] Available at: https:/www.iaras.org/iaras/home/caijts/c-its-communication-an-insight-on-the-current-research-activities-in-the-european-union [Accessed: 01 July 2018]

Martin, H., Tschabuschnig, K., Bridal, O., Watzenig, D. (2017) "Functional Safety of Automated Driving Systems: Does ISO 26262 Meet the Challenges?", In: Automated Driving, Springer, Cham, Switzerland, pp. 387-416. https://doi.org/10.1007/978-3-319-31895-0_16

Mikušová, M., Torok, A., Brída, P. (2018) "Technological and Economical Context of Renewable and Non-renewable Energy in Electric Mobility in Slovakia and Hungary", In: International Conference on Computational Collective Intelligence, Bristol, UK, pp. 429-436. https://doi.org/10.1007/978-3-319-98446-9_40

Piatkowski, B., Maciejewski, M. (2013) "Comparison of traffic assignment in VISUM and transport simulation in MATSim", Transport Problems, 8(2), pp. 113-120.

Rafat, M., Samiee, S., Azadi, S., Eichberger, A. (2019) "A Novel Real Time Flexible Lane Change Method (RFLC) in Complicated Dynamic Environment for Automated Vehicles", Vehicle System Dynamics.

Rizvi, S., Willet, J., Perino, D., Marasco, S., Condo, C. (2017) "A Threat to Vehicular Cyber Security and the Urgency for Correction", Procedia Computer Science, 114, pp. 100-105. https://doi.org/10.1016/j.procs.2017.09.021

Rosenberger, P., Holder, M., Zirulnik, M., Winner, H. (2018) "Analysis of Real World Sensor Behavior for Rising Fidelity of Physically Based Lidar Sensor Models", In: 2018. IEEE Intelligent Vehicles Symposium (IV), Changshu, China, pp. 611-616. https://doi.org/10.1109/ivs.2018.8500511

Schramm, D., Hiller, M., Bardini, R. (2018) "Equations of Motion of Complex Multibody Systems", In: Vehicle Dynamics, Springer, Berlin, Heidelberg, Germany, pp. 73-93. https://doi.org/10.1007/978-3-662-54483-9_4 
Shoup, D. C. (2006) "Cruising for parking", Transport Policy, 13(6), pp. $479-486$.

https://doi.org/10.1016/j.tranpol.2006.05.005

Sipos, T. (2017) "Spatial Statistical Analysis of the Traffic Accidents", Periodica Polytechnica Transportation Engineering, 45(2), pp. 101-105.

https://doi.org/10.3311/PPtr.9895

Szíjj, A., Buttyán, L., Szalay, Zs. (2015) "Hacking cars in the style of Stuxnet, Hacktivity", [pdf] Available at: http://www.hit.bme. hu/ buttyan/publications/carhacking-Hacktivity-2015.pdf [Accessed: 01 July 2018]

Tettamanti, T., Varga, I., Szalay, Z. (2016) "Impacts of Autonomous Cars from a Traffic Engineering Perspective", Periodica Polytechnica Transportation Engineering, 44(4), pp. 244-250. https://doi.org/10.3311/PPtr.9464

Török, Á., Szalay, Zs., Uti, G., Verebélyi, B. (2019) "Modelling the Effects of Certain Cyber-attack Methods on Urban Autonomous Transport Systems, case study of Budapest", Journal of Ambient Intelligence and Humanized Computing, 10(1), pp. 1-15. https://doi.org/10.1007/s12652-019-01264-8
Wardrop, J. G. (1952) "Some Theoretical Aspects of Road Traffic Research", Road Paper, 36, pp. 325-362.

Ye, L., Yamamoto, T. (2018) "Modeling connected and autonomous vehicles in heterogeneous traffic flow", Physica A: Statistical Mechanics and its Applications, 490, pp. 269-277. https://doi.org/10.1016/j.physa.2017.08.015

Zhao, D., Huang, X., Peng, H., Lam, H., LeBlanc, D. J. (2018) "Accelerated Evaluation of Automated Vehicles in Car-Following Maneuvers", IEEE Transactions on Intelligent Transportation Systems, 19(3), pp. 733-744. https://doi.org/10.1109/TITS.2017.2701846

Zöldy, M. (2018) "Legal Barriers of Utilization of Autonomous Vehicles as Part of Green Mobility", In: International Congress of Automotive and Transport Engineering, Cluj-Napoca, Romania, pp. 243-248.

https://doi.org/10.1007/978-3-319-94409-8_29

Zöldy, M., Kürti, C., Egresits, G. (2017) "Joint Optimization of Retail Station Operation and Secondary Distribution with Utilization of New Business Models", In: 22nd World Petroleum Congress. World Petroleum Congress, Istanbul, Turkey, pp. 34-38. 